\title{
Immunocytochemical localization of RNA polymerase I in the fibrillar centers of nucleoli
}

\author{
U. Scheer ${ }^{1}$ and I. Raska ${ }^{2}$
}

'Institute of Cell and Tumor Biology, German Cancer Research Center, D-6900 Heidelberg, Federal Republic of Germany, and ${ }^{2}$ Institute of Experimental Medicine, Czechoslovakian Academy of Sciences, Prague, CSSR

For an understanding of the functional organization of the nucleolus identification of the site of the expression of the rRNA genes is of considerable importance. Although various stages of preribosome formation have been mapped to morphologically distinct components of the nucleolus (Hadjiolov, 1985), the intranucleolar location of transcriptionally active rRNA genes has remained uncertain. From high resolution autoradiographic studies, following pulse labelling of cells with radioactive RNA precursors, it was suggested that transcription of rRNA genes takes place in the "dense fibrillar component", and possibly also at the periphery of the "fibrillar centers" (Fakan, 1978; Thiry et al., 1985). However, using other techniques such as autoradiography with tritiated thymidine or actinomycin $D$, in situ-hybridization with radioactive rRNA probes and selective DNA-staining with the osmium-amine Feulgen-like reaction, DNA (including rDNA) could be detected only in the fibrillar centers (for reviews see Hernandez-Verdun, 1983; Goessens, 1984). To reconcile these seemingly contrasting results it was proposed that the fibrillar centers contain inactive rDNA in a relatively high packing density, thereby facilitating its detection, whereas the transcribed rRNA genes loop out into the surrounding dense fibrillar component (Stahl, 1982; Goessens, 1984). According to this concept the dense fibrillar component is formed by the superposition of rRNA-transcription units and newly formed rRNP material.

Recently, we took an alternative approach to locate transcriptio- 
nally active rRNA genes within the nucleolus which was based on immunocytochemical techniques using antibodies specific for RNA polymerase I (Scheer and Rose, 1984). The results indicated that active rRNA genes are specifically confined to the fibrillar centers of rat liver nucleoli. However, since the "pre-embedding" technique used in that study may result in artifacts of selective "masking", i.e. differential accessibility of the various nucleolar components to the antibodies, we have extended these studies to antibody labelling on ultrathin frozen sections of cultured mammalian cells.

\section{IMMUNOFLUORESCENCE MICROSCOPY}

Rabbit antibodies to RNA polymerase I (RP I) stain the nucleoli of cultured cells derived from various species (Figs. $1 \mathrm{a}, 2 \mathrm{~b}, \mathrm{c}$ and $3 \mathrm{~b}$; for details see Scheer and Rose, 1984). The nucleolar fluorescence of rat cells shown in Figure 1 appears as a punctate pattern, indicating that the RP I is dispersed in several distinct subnucleolar entities (Fig. 1a; nucleoli of this cell type contain a large number of small fibrillar centers as seen in the electron microscope). A different immunofluorescence pattern is observed in cells that posess only a few, but relatively large fibrillar centers such as mouse Ehrlich ascites cells as demonstrable by electron microscopy (Fig. 2a). When these cells are grown on coverslips or processed as frozen sections of cell pellets and examined by immunofluorescence microscopy using antibodies to RP I, the staining appears in a small number (usually 2-3) of relatively large, distinct intranucleolar entities (Fig. $2 \mathrm{~b}, \mathrm{c}$ ). The homogeneous immunostaining suggests that the RP I-molecules are distributed throughout the fibrillar centers and are not confined to the peripheral layer of the dense fibrillar material.
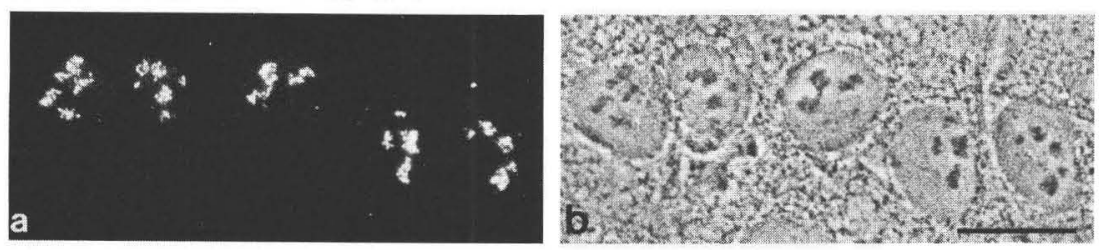

FIGURE 1. Immunofluorescence microscopy of cultured rat cells (RVFSMC, for refs. see Scheer and Rose, 1984) after staining with antibodies to RPI. Note the numerous intranucleolar dot-like substructures (a). The corresponding phase-contrast micrograph is shown in b. Bar $=20 \mu \mathrm{m}$. 

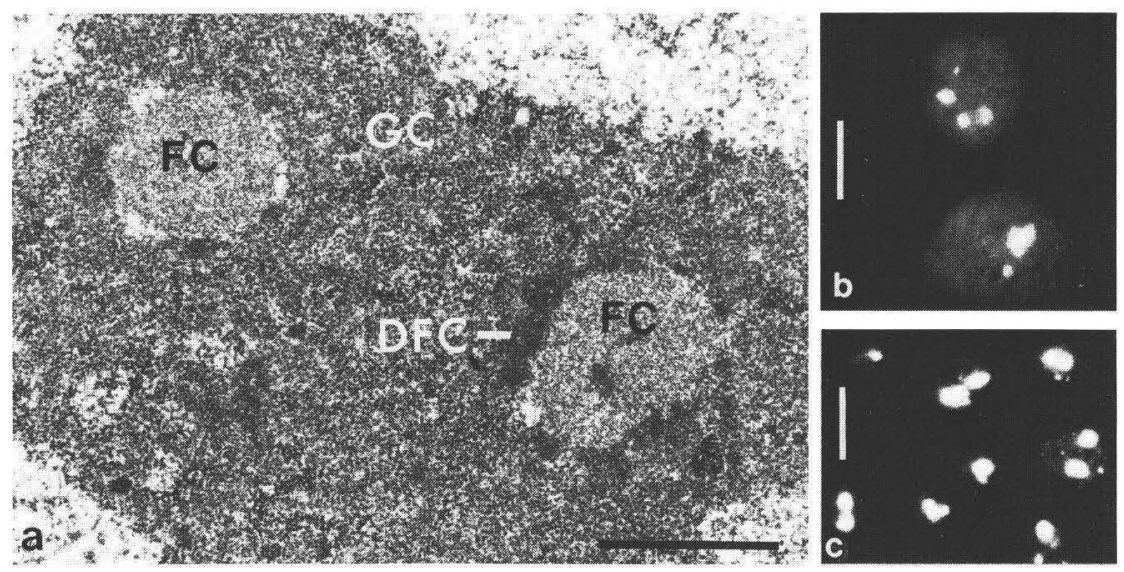

FIGURE 2. (a) Electron microscopy of a sectioned mouse cell (Ehrlich ascites) showing two large fibrillar centers (FC) embedded in the granular component of the nucleolus (GC). The dense fibrillar component (DFC) forms a thin layer around the fibrillar centers. $(b, c)$ Immunofluorescence microscopy of Ehrlich cells grown on coverslips (b) and of a cryostat section through a cell pellet (c) after incubation with anti-RP I. The nucleoli contain large fluorescent spheres. Bars $=1 \mu \mathrm{m}(\mathrm{a}) ; 10 \mu \mathrm{m}(\mathrm{b}, \mathrm{c})$.

Nucleoli of cultured Xenopus cells also contain relatively large fibrillar centers (Fig. 3a). Correspondingly, immunofluorescence microscopy with antibodies to RP I reveals intranucleolar aggregates of distinctly fluorescing spheres (Fig. 3b).

\section{ELECTRON MICROSCOPIC CHROMATIN SPREAD PREPARATIONS}

Very brief exposure of cells to low salt buffer, prior to centrifugation onto electron microscopic grids, retain to some extent the original spatial arrangement of the rRNA genes, although in such preparations the topological organization of the various nucleolar components is usually lost. As shown in Figure 3d, distinct roundish clusters each containing 10 to 30 closely packed and intenseIy transcribed rRNA genes are observed when chromatin of cultured Xenopus cells is gently spread for electron microscopy.

ACTINOMYCIN D (AMD)-INDUCED REDISTRIBUTION OF RNA POLYMERASE I

Inhibition of transcription by exposure of Xenopus cells to AMD leads to a rearrangement of the fibrillar centers and the other nucleolar components ("nucleolar segregation"). Typically the fibrillar centers appear in a more peripheral location, partly surrounding the segregated dense fibrillar component (Fig. 3e). This rearrange- 

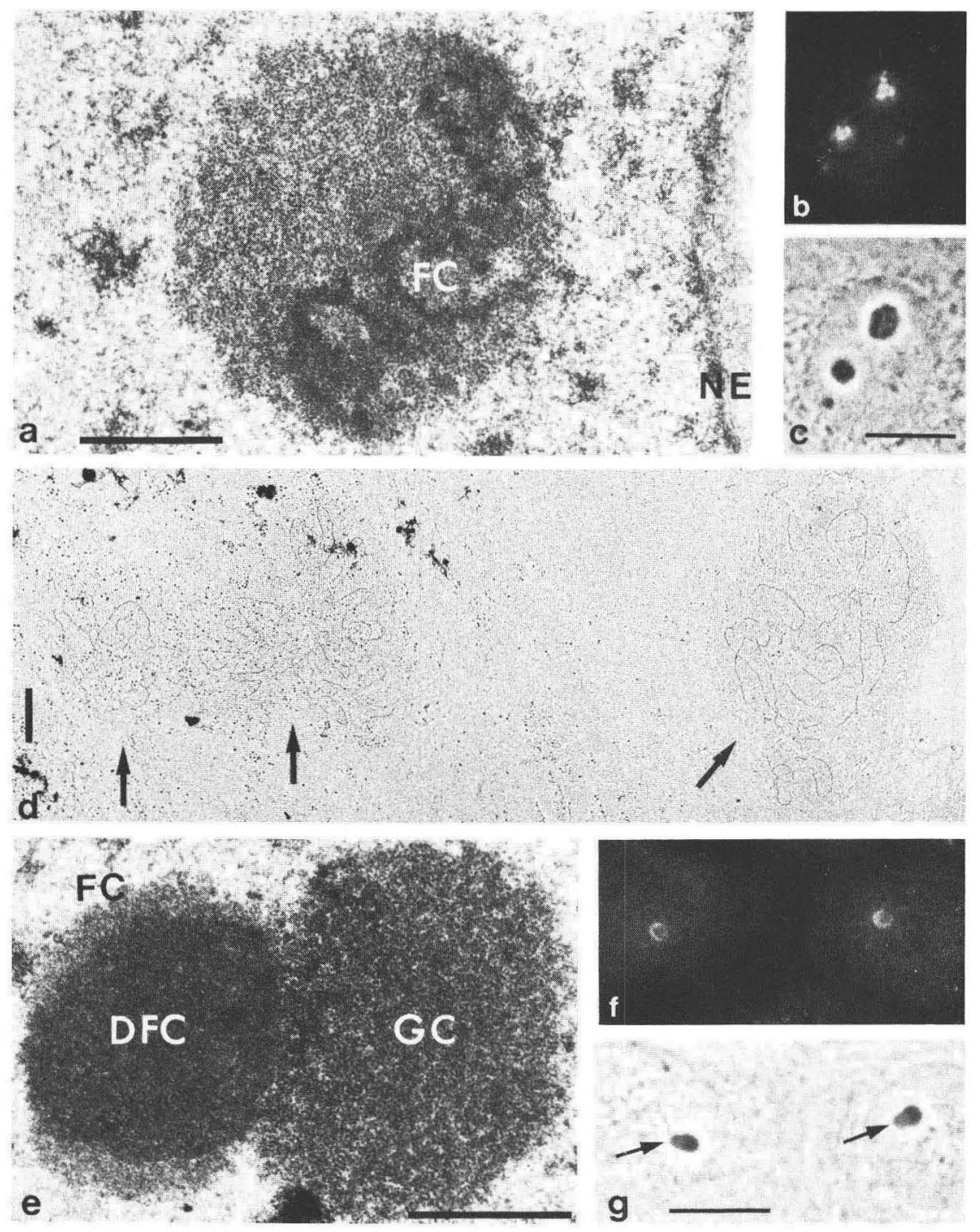

FIGURE 3. Cultured Xenopus laevis kidney epithelial cells (line A6; American Type Culture Collection). (a) Electron microscopy reveals the three nucleolar components (see Fig. 2). NE, nuclear envelope. (b,c) Immunofluorescence microscopy (c, phase contrast) after staining with anti-RP I. (d) Chromatin spread preparation after very brief lysis of Xenopus cells in low salt buffer (for technical details see Scheer and Zentgraf, 1982). Active rRNA genes occur in several compact aggregates (arrows). (e) Electron microscopy (ultrathin section) of an segregated nucleolus after $4 \mathrm{~h}$ of AMD-treatment $(5 \mu \mathrm{g} / \mathrm{ml})$. ( $f, g)$ Immunofluorescence microscopy of AMD-treated Xenopus cells after staining with anti-RP I. The fluorescent cap (f) is situated on top of the phase-light dense fibrillar component (arrows in $g$ ). Bars $=1 \mu \mathrm{m}(a, d, e) ; 10 \mu \mathrm{m}(c, g)$. 
ment of the nucleolar components upon AMD-treatment results in a considerable decrease in immunostaining intensity and a redistribution of the RP I-containing structures from an intranucleolar (Fig. 3b) to a peripheral (Fig. 3f) position. The crescent-shaped RP I-positive component of segregated nucleoli always surrounds the dense fibrillar component (Fig. $3 f, g)$.

\section{IMMUNOGOLD ELECTRON MICROSCOPY}

The "pre-embedding" labelling technique as described previously (Scheer and Rose, 1984) has the advantage that the antibody binding reaction can be performed on frozen tissue sections (about $5 \mu \mathrm{m}$ thick) without any prior chemical fixation. For example, in Figure 4 individual nucleolar components of a rat hepatocyte are clearly recognized. Gold particles, reflecting the distribution of the bound RP I antibodies, are enriched over the fibrillar centers and are clearly absent from the dense fibrillar component.

Immunolabelling of ultrathin frozen sections ("post-embedding" technique) gives essentially identical results. Figure 5 shows an ultrathin cryosection of fixed ( $4 \%$ paraformaldehyde) cultured PC12 rat cells incubated with RP I antibodies, followed by secondary

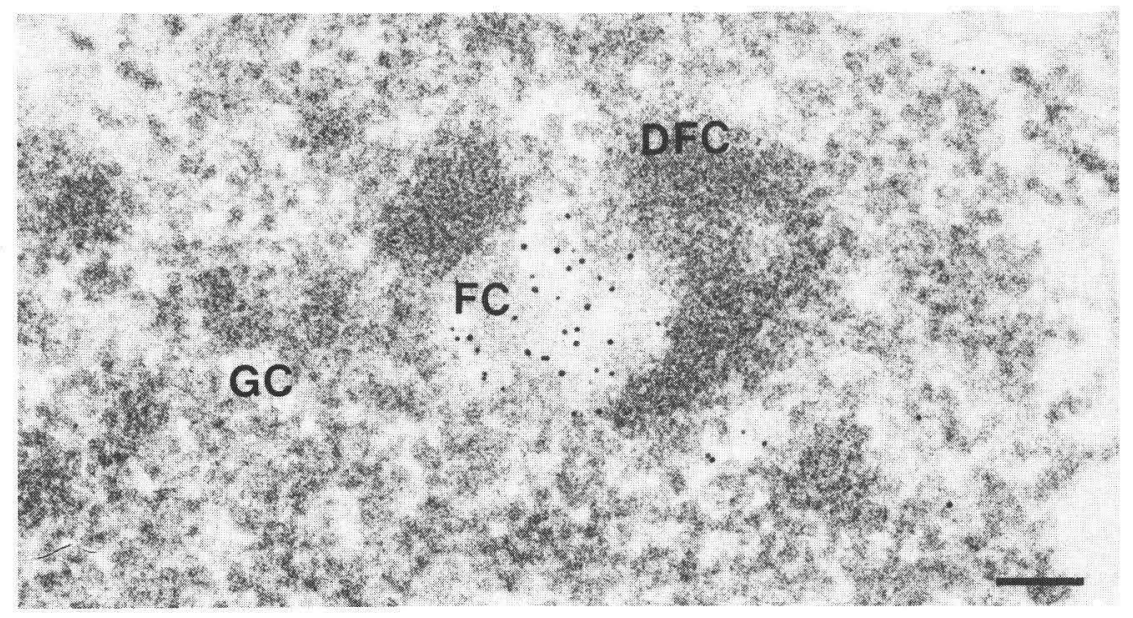

FIGURE 4. Electron microscopic immunolocalization of RP I in rat hepatocytes. Cryostat sections were incubated with antibodies to RP I followed by secondary antibodies coupled to $5 \mathrm{~nm}$ gold particles. After the immunoreaction the tissue section was processed for electron microscopy (see Scheer and Rose, 1984). Gold particles are selectively enriched over the fibrillar center (FC). Bar = $0.1 \mu \mathrm{m}$. 


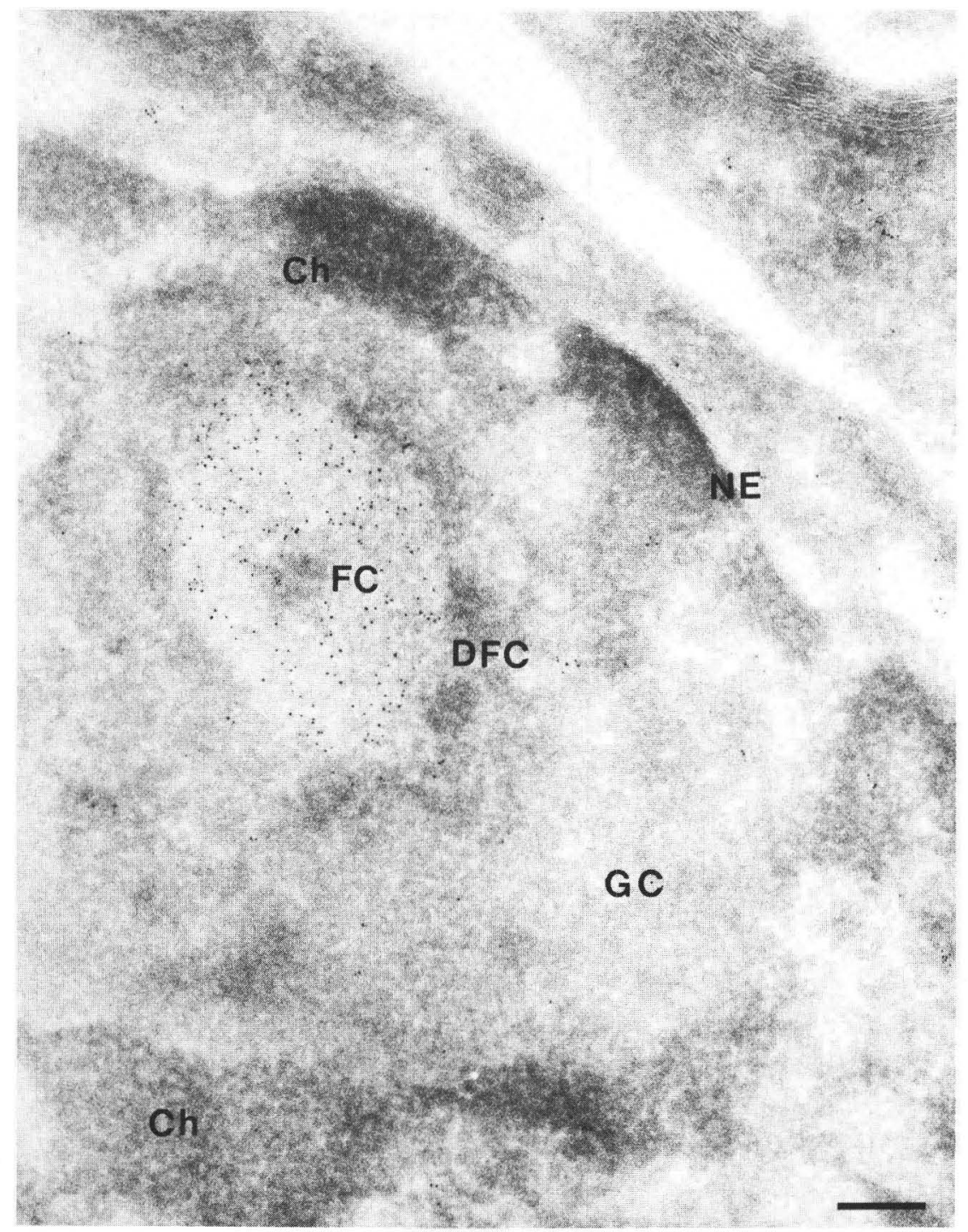

FIGURE 5. RP I antibody labelling of an ultrathin frozen section of cultured rat PC12 cells (Greene and Tischler, 1976). A cell pellet was fixed in 4\% formaldehyde for $60 \mathrm{~min}$, washed in PBS, incubated in $2.1 \mathrm{M}$ sucrose and shock-frozen in liquid nitrogen. Cryosections were cut and incubated with antibodies essentially as described by Tokuyasu (1980). Secondary antibodies coupled to $5 \mathrm{~nm}$ gold particles were purchased from Janssen Life Sciences (Beerse, Belgium) and used in a 1:10 dilution. Gold particles are enriched over the large fibrillar center (FC) and are almost absent from the surrounding dense fibrillar component (DFC) and the granular component (GC). Ch, nucleolus-associated heterochromatin; NE, nuclear envelope. Bar $=0.2 \mu \mathrm{m}$. 
antibodies coupled to collodial gold. The gold particles are selectively enriched over the large fibrillar center. All other nucleolar structures are not significantly labelled above background. Human autoantibodies to RP I, which occur in sera of scleroderma patients, also decorate specifically the fibrillar centers of these cells (Fig. 6; for details see Reimer et al., 1986). Again, there is no significant enrichment of gold particles in the dense fibrillar component.

\section{CONCLUSIONS}

The present results confirm and extend our previous conclusion based on a "pre-embedding" immunocytochemical approach that RP I is found exclusively in the fibrillar centers of nucleoli (Scheer and Rose, 1984). Does this result allow the conclusion that transcription of rDNA primarily or even exclusively takes place in this nucleolar compartment? It could be argued, for instance, that the binding of RP I antibodies to the fibrillar centers merely reflects the presence of a pool of "stored" enzymes bound to an unknown structure and that the transcribing polymerases are in fact present in the dense fibrillar component but not accessible to the antibodies. Moreover, the antibodies could recognize only a specific posttranslational modification present in inactive RP I but absent in transcribing molecules. However, several arguments speak against this view which can be summarized as follows.

1. The absence of antibody labelling in the dense fibrillar component most likely reflects the absence of RNA polymerase I. Antibody labelling on ultrathin frozen sections provides optimal accessibility of epitopes. Thus, most likely, the absence of gold label over the dense fibrillar component is not due to a selective "masking" effect. Another possibility, i.e. selective recognition of free vs. template-engaged RP I also seems unlikely since the rabbit antibodies used contain IgGs directed against epitopes of most of the RP I-subunits (Rose et al., 1981).

2. Antibody binding to the fibrillar centers is not due to a storage pool of polymerases. It is well known that RP I as well as the other RNA polymerase classes exist in two functionally distinct states, i.e. template-bound and free (for reviews see Grummt, 1978; Muramatsu et al., 1979). The free form of RP I is readily diffusible 


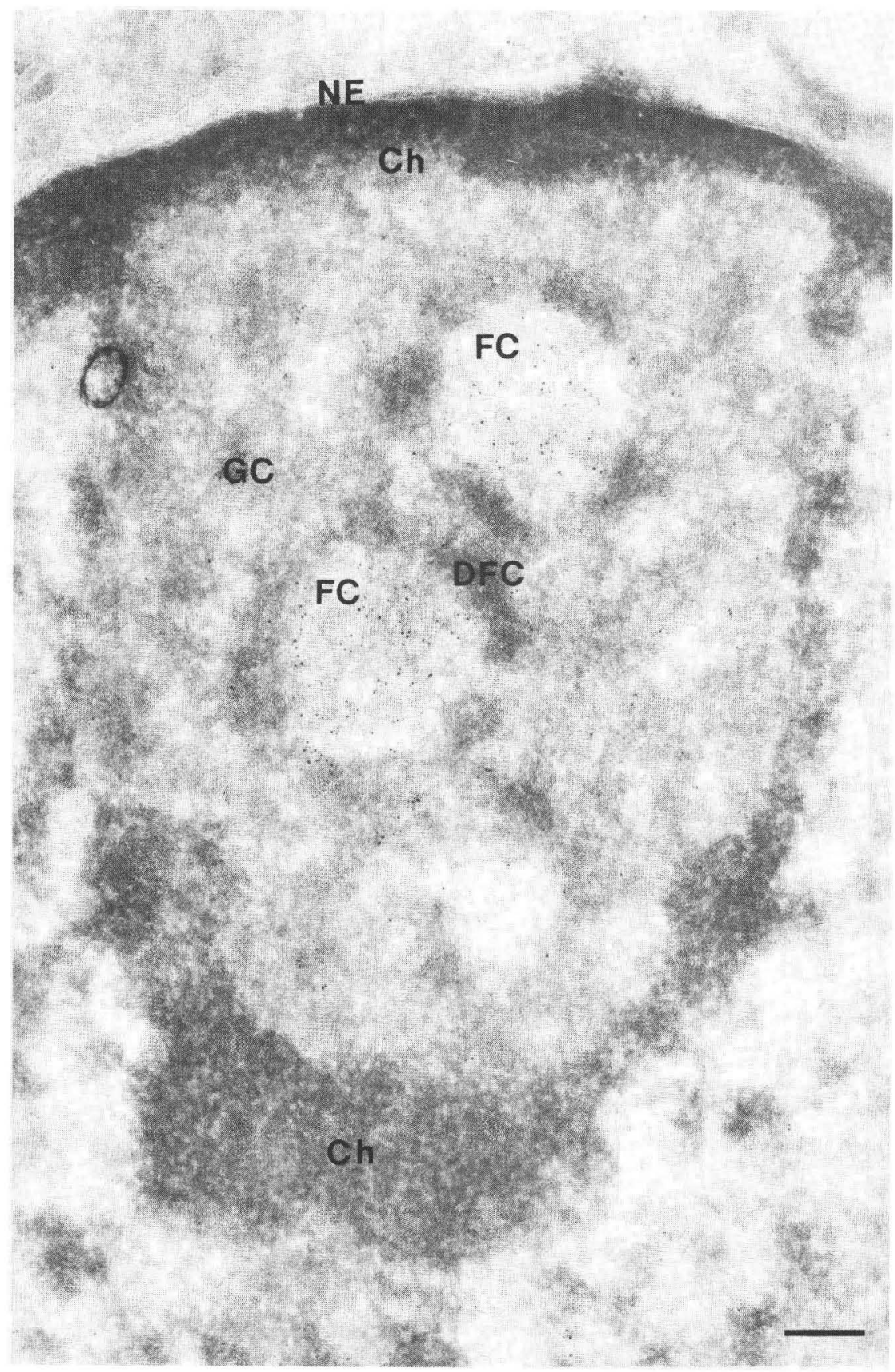

FIGURE 6. Similar preparation as shown in Fig. 5 but after incubation with an autoimmune serum from a scleroderma patient with anti-RP I specificity (Reimer et al., 1986). Again, gold particles are concentrated in the fibrillar centers $(\mathrm{FC})$. Bar $=0.2 \mu \mathrm{m}$. 
and released from isolated nuclei upon incubation in isotonic buffers (about 20\% of rat liver RP I exists in the free form; Yu, 1980; see also Jacob and Rose, 1978). Furthermore, the observation that addition of exogenous DNA templates to nucleoli isolated under conditions minimizing RP I leakage stimulates RNA synthesis, also argues against a stable association of the free RP I to nucleolar structures (see Grummt, 1978; Muramatsu et al., 1979). That the soluble pool of RP I does not contribute to the present immunolocalization results is demonstrated by a considerable reduction in nucleolar immunostaining following treatment of cells with AMD. Short-term exposure of cells to AMD does not change the total amount of RP I-molecules present but shifts the equilibrium between free and engaged enzymes toward the free form.

3. Initiated but blocked polymerases are not detectable in nucleoli. This "frozen" status of RNA polymerases is detected when transcription of nuclei, chromatin or isolated chromosomes is assayed in vitro under conditions excluding new initiation events ("run-on" or "elongation"-assay). Stimulation of transcription by removal of DNAassociated proteins with the polyanion heparin or the anionic detergent Sarkosyl indicates the presence of pre-existing but blocked transcriptional complexes. When isolated nuclei were assayed with or without heparin, no significant stimulation of RP I-dependent transcription was observed (Coupar and Chesterton, 1977). Thus, it can be excluded that the polymerases present in the fibrillar centers represent a special form of initiated but transcriptionally inactive enzymes. A different situation is encountered during mitosis. RP I remains bound to the nucleolus organizing regions (NOR) of mitotic chromosomes (Scheer and Rose, 1984), apparently as initiated complexes with rDNA which, however, do not elongate (Matsui and Sandberg, 1985).

Taken together, we feel that our immunocytochemical data justify the conclusion that transcription of rRNA genes takes place in the fibrillar centers of nucleoli. As mentioned in the Introduction, this conclusion agrees with several studies showing that DNA including rDNA is present in the fibrillar centers but is at variance with certain autoradiographic studies of RNA labelling. However, it has to be kept in mind that pulse-labelling of cells with radioactive uridine necessarily leads to two different states of radioac- 
tive RNAs, i.e. template-attached and released molecules. Furthermore, a labelling period of 2-5 min which is often used (Fakan, 1978; Stahl, 1982; Goessens, 1984; Thiry et al., 1985) is about the time needed for an individual RP I molecule to transcribe an entire rRNA gene (approximately 3-5 min). Immediate translocation of the completed pre-rRNAs to and accumulation in the dense fibrillar component would lead to a high local packing density of RNA in this compartment. Thus, the distribution of silver grains might reflect primarily the site where pre-rRNAs accumulate after their release from the template rather than the site of transcription.

We trust that the precise location of transcribing rRNA genes within the nucleolus will enable us, by using a series of antibodies to different ribosomal and nucleolus-specific proteins, to understand in more detail the spatial and temporal patterns of interaction between pre-rRNAs and proteins involved in the formation of nucleolar preribosomal particles.

Acknowledgements: We thank Drs. W.W. Franke and F. Longo for their critical reading of the manuscript. We are grateful to Dr. K. Rose (University of Texas, Houston, Texas) for rabbit antibodies to RP I, Drs. G. Reimer and E. Tan (Scripps Clinic, La Jolla, California) for human autoimmune sera, and Dr. C. Granzow (this institution) for Ehrlich ascites cells. This work was supported by grants from the Deutsche Forschungsgemeinschaft (Sche 157/5-4).

\section{REFERENCES}

Coupar, B.E.H. and C.J. Chesterton. 1977. The mechanism by which heparin stimulates transcription in isolated rat liver nuclei. Eur. J. Biochem. 79, 525-533.

Fakan, S. 1979. High resolution autoradiography studies on chromatin functions. In The cell nucleus, vol. 5, H. Busch, ed., 3-53. New York: Academic Press.

Goessens, G. 1984. Nucleolar structure. Int. Rev. Cytol. 87, 107-158.

Greene, L.A. and A.S. Tischler. 1976. Establishment of a noradrenergic clonal line of rat adrenal pheochromocytoma cells which respond to nerve growth factor. Proc. Natl. Acad. Sci. USA 73, 2424-2428.

Grummt, I. 1978. In vitro synthesis of pre-rRNA in isolated nucleoli. In The cell nucleus, vol. 5, H. Busch, ed., 313-414. New York: Academic Press.

Hadjiolov, A.A. 1985. The nucleolus and ribosome biogenesis. Vienna: Springer-Verlag.

Hernandez-Verdun, D. 1983. The nucleolar organizer region. Biol. Cell. 49; 191-202. 
Jacob, S.T. and K.M. Rose. 1978. RNA polymerases and poly (A) polymerase from neoplastic tissues and cells. In Methods in cancer research, vol. 14, H. Busch, ed., 191-241. New York: Academic Press.

Matsui, S. and A.A. Sandberg. 1985. Intranuclear compartmentalization of DNA-dependent RNA polymerases: association of RNA polymerase I with nucleolar organizing chromosomes. Chromosoma $92,1-6$.

Muramatsu, M., T. Matsui, T. Onishi and Y. Mishima. 1979. Nucleolar RNA polymerase and transcription of nucleolar chromatin. In The cell nucleus, vol. 7, H. Busch, ed., 123-161. New York: Academic Press.

Reimer, G., K.M. Rose, U. Scheer and E. Tan. 1986. Autoantibody to RNA polymerase I in scleroderma sera. Submitted to J. clin. Invest.

Rose, K.M., D.A. Stetler and S.T. Jacob. 1981. Protein kinase activity of RNA polymerase I purified from a rat hepatoma: probable function of $\mathrm{M}_{\mathrm{r}} 42,000$ and 24,600 polypeptides. Proc. Natl. Acad. Sci. USA 78, ${ }^{\mathrm{Y}} 2833-2837$.

Scheer, U. and H. Zentgraf. 1982. Morphology of nucleolar chromatin in electron microscopic spread preparations. In The cell nucleus, vol. 11, H. Busch, ed., 143-176. New York: $\overline{\text { Academic }}$ Press.

Scheer, U. and K.M. Rose. 1984. Localization of RNA polymerase I in interphase cells and mitotic chromosomes by light and electron microscopic immunocytochemistry. Proc. Natl. Acad. Sci. USA 81, 1431-1435.

Stahl, A. 1982. The nucleolus and nucleolar chromosomes. In The nucleolus, E.G. Jordan and C.A. Cullis, eds., 1-24. Cambridge: Cambridge University Press.

Thiry, M., A. Lepoint and G. Goessens. 1985. Re-evaluation of the site of transcription in Ehrlich tumour cell nucleoli. Biol. Cell. $54,57-64$.

Tokuyasu, K.T. 1980. Immunocytochemistry on ultrathin frozen sections. Histochem. J. 12, 381-403.

Yu, F. (1980) High concentration of RNA polymerase I is responsible for the high rate of nucleolar transcription. Biochem. J. 188, 381-385. 